\title{
Rich Dynamics of an Epidemic Model with Saturation Recovery
}

\author{
Hui Wan ${ }^{1}$ and Jing-an Cui ${ }^{2}$ \\ ${ }^{1}$ Jiangsu Key Laboratory for NSLSCS, School of Mathematics, Nanjing Normal University, Nanjing 210046, China \\ ${ }^{2}$ School of Sciences, Beijing University of Civil Engineering and Architecture, Beijing 100044, China \\ Correspondence should be addressed to Jing-an Cui; cuijingan@bucea.edu.cn
}

Received 18 January 2013; Accepted 26 March 2013

Academic Editor: Xinyu Song

Copyright (C) 2013 H. Wan and J.-a. Cui. This is an open access article distributed under the Creative Commons Attribution License, which permits unrestricted use, distribution, and reproduction in any medium, provided the original work is properly cited.

\begin{abstract}
A SIR epidemic model is proposed to understand the impact of limited medical resource on infectious disease transmission. The basic reproduction number is identified. Existence and stability of equilibria are obtained under different conditions. Bifurcations, including backward bifurcation and Hopf bifurcation, are analyzed. Our results suggest that the model considering the impact of limited medical resource may exhibit vital dynamics, such as bistability and periodicity when the basic reproduction number $\mathbb{R}_{0}$ is less than unity, which implies that the basic reproductive number itself is not enough to describe whether the disease will prevail or not and a subthreshold number is needed. It is also shown that a sufficient number of sickbeds and other medical resources are very important for disease control and eradication. Considering the costs, we provide a method to estimate a suitable treatment capacity for a disease in a region.
\end{abstract}

\section{Introduction}

In recent years, efforts have been made to develop realistic mathematical models for the transmission dynamics of known and emerging infectious diseases [1-8]. The development of such models aims at understanding the epidemiological transmission patterns and predicting the consequences of the introduction of public health interventions to control the possible outbreak and spread of the disease.

In this paper, we will focus on the impact of limited medical resource or treatment capacity on infectious disease transmission. In fact, each city should have its suitable treatment capacity. If it is too large, the city pays for unnecessary costs. While if it is too small, the city has the risk of the outbreak of a disease. Statistics [9] show that, for example, there are 16.4 sickbeds for every 1000 residents in Japan on average in 1999, but the corresponding data are 2.6, 2.4, and 1.1, respectively, in Turkey, China, and Mexico. Thus, it is important to understand the impact of limited medical resource on disease transmission and to determine a suitable treatment capacity for a disease.

In classical disease transmission models, the recovery from infected class per unit of time is assumed to be proportional to the number of infective individuals. However, it is not a reasonable approximation to the truth when the number of the infectious individuals is large and the treatment capacity of hospitals is researched, provided that the infected individuals cannot recover unless they were given timely treatment in hospitals. Then the number of patients needing to be treated in hospitals may exceed the number of the hospital beds. In this case, the recovery rate from infective class will be saturated at a maximum, especially in the rural areas in many developing countries.

In paper [10], Wang and Ruan adopt a constant treatment rate, which simulates a limited capacity for treatment. Note that a constant treatment is suitable when the number of infectives is large; hence in [7], Wang modified the rate of treatment to another function which is proportional to the number of the infectives when the capacity of treatment is not reached and, otherwise, takes the maximal capacity. In order to model the impact of limited medical resource on infectious disease transmission precisely and provide a method to estimate a suitable treatment capacity for a disease, we propose an epidemic model with saturation recovery.

The organization of this paper is as follows. In Section 2, we propose an SIR epidemic model and analyze the diseasefree equilibrium. In Section 3, we mainly discuss the existence 
of endemic equilibrium (EE). In Section 4, the stability of EE and bifurcation are presented. Section 5 is the discussion.

\section{The Model}

In order to give a more realistic recovery rate for epidemic models and study the impact of limited medical resource on disease transmission, in this paper, we adopt the Verhulsttype function $([11])$

$$
h(I)=\frac{c I}{b+I}
$$

to model the treated part which is increasing for small infectives and approaches the maximum for large infectives. Hence $c$, as the limit of $h(I)$ as $I$ tends to infinity, is the maximum of the treatment capacity in a region, and $b$, the infected size at which is $50 \%$ saturation $(h(b)=c / 2)$, measures how soon saturation occurs. Here $h(I)$ satisfies $h(0)=0, h(\infty)=c$ and $d h / d I>0$, which means that the treatment rate is increasing for a small number of infectives and approaches a maximum for large number of infectives.

We classify the population in a given region/area into three categories: susceptible, infective, and recovered. Let $S(t), I(t)$, and $R(t)$ denote the number of susceptible, infective, and recovered individuals at time $t$, respectively. Provided that the infected individuals cannot recover unless they were given timely treatment in hospitals, based on standard SIR model with mass action incidence, we can construct a model

$$
\begin{gathered}
\frac{d S}{d t}=A-d S-\beta S I \\
\frac{d I}{d t}=\beta S I-(d+v) I-\frac{c I}{b+I}, \\
\frac{d R}{d t}=\frac{c I}{b+I}-d R
\end{gathered}
$$

where all the parameters are positive and

(i) $A$ is the recruitment rate of susceptible population;

(ii) $d$ is natural death rate and $v$ is the disease-induced death rate;

(iii) $c$ is the maximum of treatment per unit of time, and $b$, the infected size at which is $50 \%$ saturation $(h(b)=$ $c / 2$ ), measures how soon saturation occurs;

(iv) $\beta$ is the transmission rate.

Note that the first two equations are independent of the third one; we need only to study the following reduced model:

$$
\begin{gathered}
\frac{d S}{d t}=A-d S-\beta S I, \\
\frac{d I}{d t}=\beta S I-(d+v) I-\frac{c I}{b+I} .
\end{gathered}
$$

Model (3) has one disease-free equilibrium at $E_{0}=$ $(A / d, 0)$. Using the formulae in [12], a straightforward calculation gives the reproduction number:

$$
\mathbb{R}_{0}=\frac{A b \beta}{d(b d+b v+c)} .
$$

The disease-free equilibrium $E_{0}$ has two eigenvalues $-d$ and $\mathbb{R}_{0}-1$. Therefore we have the following proposition.

Proposition 1. For the model (3), the disease-free equilibrium $E_{0}$ is locally asymptotically stable if $\mathbb{R}_{0}<1$ and unstable if $\mathbb{R}_{0}>1$.

\section{Existence of the Endemic Equilibrium}

Let

$$
h(I)=\frac{A}{d+\beta I}, \quad g(I)=\frac{d+v}{\beta}+\frac{c}{\beta(b+I)} .
$$

Then we can rewrite the model (3) as

$$
\begin{gathered}
\frac{d S}{d t}=-(d+\beta I)(S-h(I)), \\
\frac{d I}{d t}=\beta I(S-g(I)) .
\end{gathered}
$$

Let the right hand side of (6) be zero. If an endemic equilibrium exists, its $(S, I)$ coordinates must satisfy

$$
S=g(I), \quad S=h(I) .
$$

We note that $\lim _{I \rightarrow \infty} h(I)=0, \lim _{I \rightarrow \infty} g(I)=(d+$ $\nu) / \beta, d h / d I<0, d g / d I<0$, and $d^{2} h / d I^{2}>0, d^{2} g / d I^{2}>0$. In addition, $\mathbb{R}_{0}=h(0) / g(0)$.

Eliminating $S$ from (7) gives an equation of the form

$$
I^{2}+a_{1} I+a_{2}=0
$$

where

$$
\begin{gathered}
a_{1}=\frac{(b \beta+d)(d+\nu)+c \beta-A \beta}{\beta(d+\nu)}, \\
a_{2}=\frac{b d(d+v)+c d-\beta A b}{\beta(d+v)}=\frac{b d(d+v)+c d}{\beta(d+\nu)}\left(1-\mathbb{R}_{0}\right) .
\end{gathered}
$$

If $\mathbb{R}_{0}>1$, then $a_{2}<0$, and there is a unique positive root for (8) which implies that a unique endemic equilibrium $E^{*}\left(S^{*}, I^{*}\right)$ exists (see Figure $1(\mathrm{~b})$ ).

If $\mathbb{R}_{0}=1$, then $a_{2}=0$ and there is a unique nonzero solution of (8) $I=-a_{1}$ which is positive if and only if $a_{1}<0$. Then, there is a unique endemic equilibrium $E^{*}\left(S^{*}, I^{*}\right)$ when $a_{1}<0$, and there are not endemic equilibria when $a_{1} \geq 0$.

If $\mathbb{R}_{0}<1$, then $a_{2}>0$. For (8) to have at least one positive root we must have

$$
a_{1}<0, \quad \Delta \triangleq a_{1}^{2}-4 a_{2} \geqslant 0 .
$$




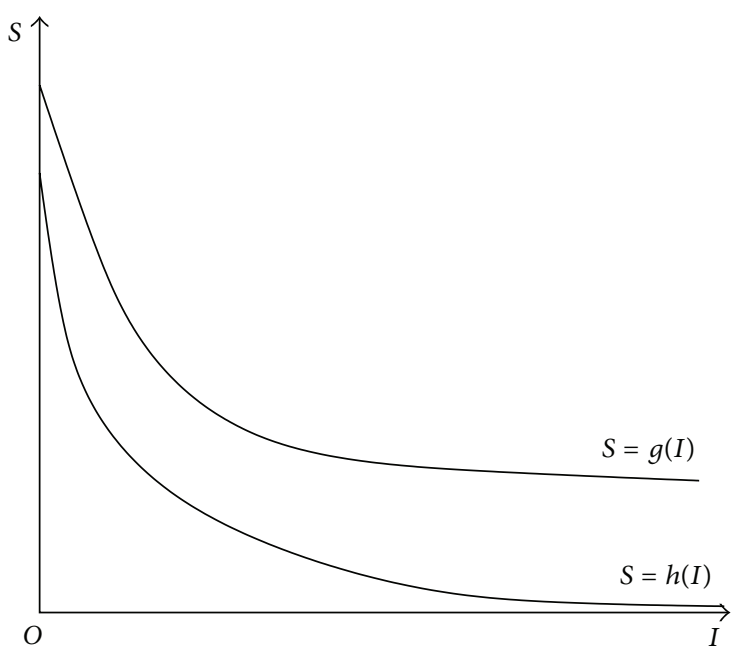

(a) No positive equilibrium

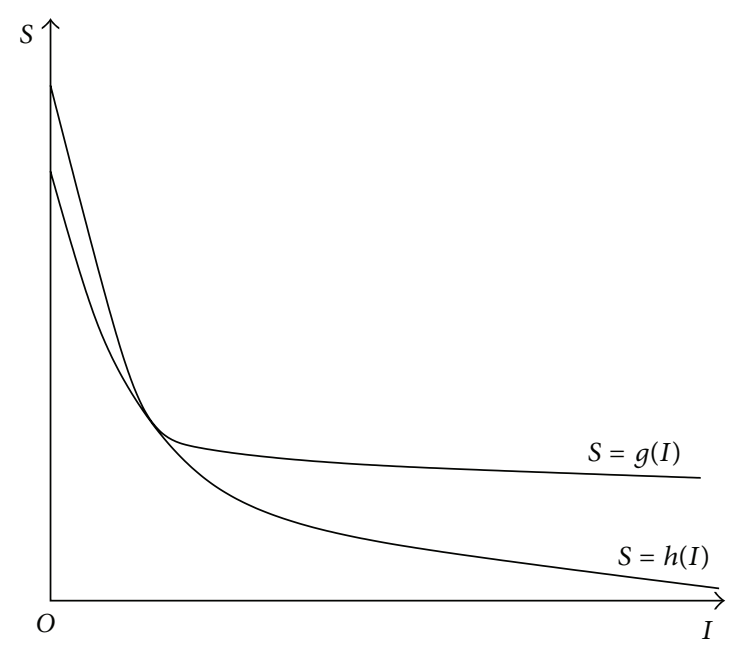

(c) Unique equilibrium of multiplicity 2

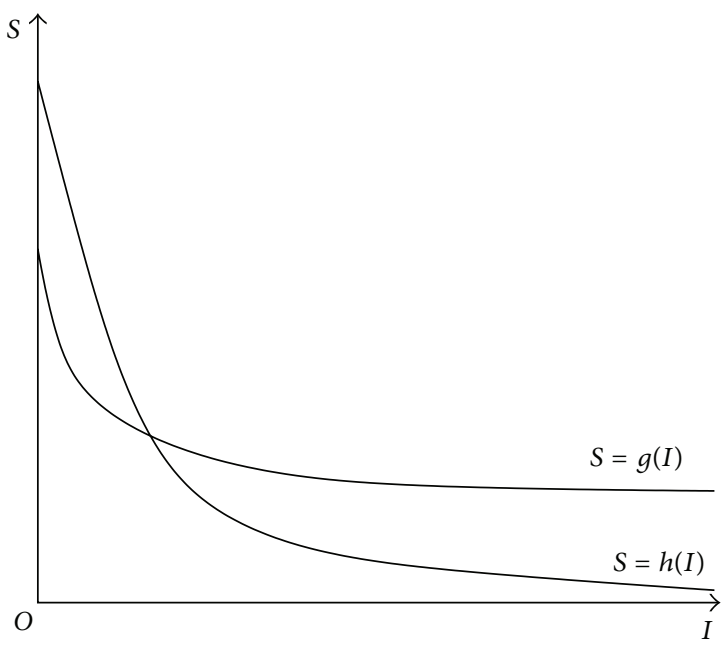

(b) Unique positive equilibrium

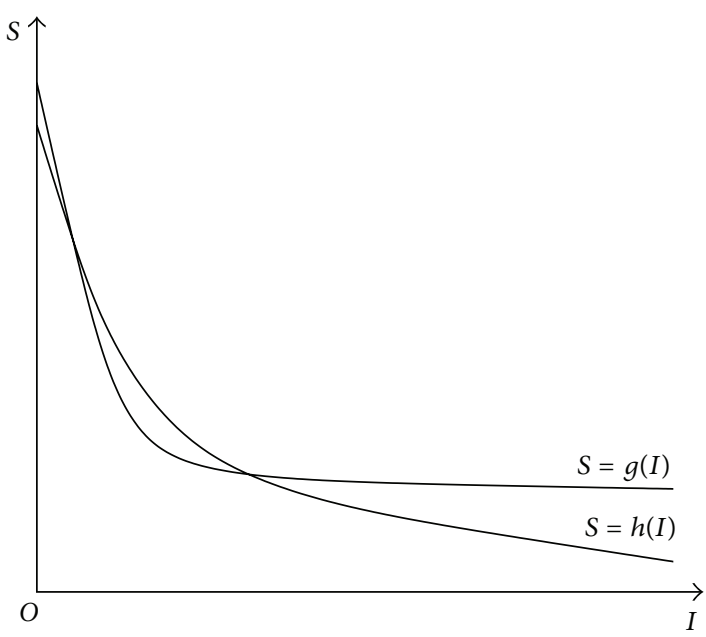

(d) Two positive equilibria

FIGURE 1: Existence and number of endemic equilibria.

Solving $\Delta=0$ in terms of $\mathbb{R}_{0}$, one gets $\mathbb{R}_{0}=\widehat{\mathbb{R}}_{0}$ where

$$
\begin{gathered}
\widehat{\mathbb{R}}_{0}(c) \\
=A b(d+v)\left[b(d+v)+(\sqrt{A}+\sqrt{c})^{2}\right] \\
\times((c+b(d+v))[b(d+v)(b(d+v)+2(A+c)) \\
\left.\left.+(A-c)^{2}\right]\right)^{-1} .
\end{gathered}
$$

One can verify that, provided $a_{1}<0$, model (3) has exactly 0 , 1, and 2 endemic equilibria as shown in Figures 1(a), 1(c), and $1(\mathrm{~d})$ for $\mathbb{R}_{0}<\widehat{\mathbb{R}}_{0}, \mathbb{R}_{0}=\widehat{\mathbb{R}}_{0}$, and $\mathbb{R}_{0}>\widehat{\mathbb{R}}_{0}$, respectively.

In summary, regarding the existence of endemic equilibrium, we have the following.
Theorem 2. For model (3), one has the following.

(1) If $\mathbb{R}_{0}>1$, there exists a unique positive equilibrium $E^{*}\left(S^{*}, I^{*}\right)$.

(2) If $\mathbb{R}_{0}=1$; there is a positive equilibrium $E^{*}\left(S^{*}, I^{*}\right)$ when $a_{1}<0$; otherwise there is no positive equilibrium.

(3) If $\mathbb{R}_{0}<1$ and $a_{1} \geq 0$, there is no positive equilibrium.

(4) If $\widehat{\mathbb{R}}_{0}<\mathbb{R}_{0}<1$ and $a_{1}<0$, there are two positive equilibria $E^{*}$ and $E_{*}$.

(5) If $\mathbb{R}_{0}=\widehat{\mathbb{R}}_{0}$ and $a_{1}<0, E^{*}$ and $E_{*}$ coalesce together as a unique equilibrium of multiplicity two.

(6) If $\mathbb{R}_{0}<\widehat{\mathbb{R}}_{0}$ and $a_{1}<0$, there is no positive equilibrium. 
When exist, $E^{*}\left(S^{*}, I^{*}\right)$ and $E_{*}\left(S_{*}, I_{*}\right)$ are the corresponding equilibria, and

$$
\begin{array}{cc}
S^{*}=h\left(I^{*}\right), & S_{*}=h\left(I_{*}\right), \\
I^{*}=\frac{-a_{1}+\sqrt{\Delta}}{2}, & I_{*}=\frac{-a_{1}-\sqrt{\Delta}}{2} .
\end{array}
$$

$c$, the maximum treatment per unit of time, is related to the treatment capacity in a region and the recruitment rate of susceptible individuals $A$ can be changed by vaccination, and so forth; they are all important for disease control. In the following section, we choose $c$ and $A$ as parameters to discuss the distribution of the equilibria of system (6) in plane $(c, A)$. Solving equations $a_{1}=0$ and $a_{2}=0$ for $A$, we can get $A=$ $A_{1}(c)$ and $A=A_{2}(c)$, respectively, where

$$
\begin{gathered}
A_{1}(c)=c+\left(b+\frac{d}{\beta}\right)(d+v), \\
A_{2}(c)=\frac{d}{b \beta} c+\frac{d(d+\nu)}{\beta} .
\end{gathered}
$$

Then

$$
\begin{aligned}
& a_{1}=\left(A_{1}(c)-A\right) \frac{1}{d+v}, \\
& a_{2}=\left(A_{2}(c)-A\right) \frac{b}{d+v} .
\end{aligned}
$$

Obviously, $a_{1}>0$ (or $a_{1}<0$ ) when $A<A_{1}(c)$ (or $A>$ $\left.A_{1}(c)\right)$ and $a_{2}>0\left(\right.$ or $\left.a_{2}<0\right)$ when $A<A_{2}(c)\left(\right.$ or $\left.A>A_{2}(c)\right)$.

It is easy to see that if $A>A_{2}(c),(7)$ has a unique positive solution.

Now we consider the case when $A \leqslant A_{2}(c)$ (the case $a_{2} \geq$ $0)$. Writing the discriminant $\Delta=a_{1}^{2}-4 a_{2}$ as a function of $c$ and $A$, we have

$$
\begin{aligned}
\Delta(c, A)= & {\left[\frac{(b \beta+d)(d+\nu)+c \beta-A \beta}{\beta(d+\nu)}\right]^{2} } \\
& -4\left[\frac{b d(d+\nu)+c d-\beta A b}{\beta(d+\nu)}\right] .
\end{aligned}
$$

If $\Delta=0$ defines curves in the first quadrant of the $(c, A)$ plane, then it must satisfy $A \leqslant A_{2}(c)$. Furthermore, if (8) has nonnegative roots, we need $A_{1}(c)<A \leqslant A_{2}(c)$ and $\Delta \geqslant 0$. Calculate the difference of $A_{2}(c)$ and $A_{1}(c)$ :

$$
A_{2}(c)-A_{1}(c)=\frac{d-b \beta}{b \beta} c-b(d+v) .
$$

If $d-b \beta \leqslant 0$, then $A_{2}(c)<A_{1}(c)$ and system (6) does not have any endemic equilibrium when $A \leqslant A_{2}(c)$.

If $d-b \beta>0$, one easily gets that $c=c^{*}$ from $A_{2}(c)-$ $A_{1}(c)=0$, where

$$
c^{*}=\frac{b^{2} \beta(d+\nu)}{d-b \beta} .
$$

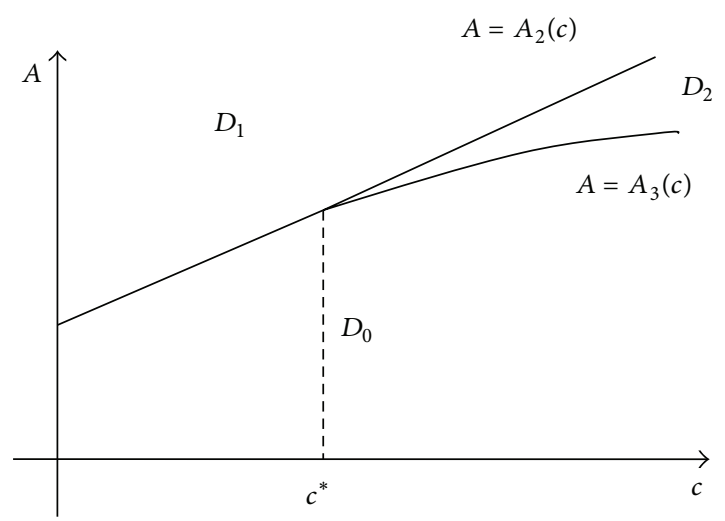

FIgURE 2: The distribution of equilibrium on the plane of $(c, A)$ when $d-b \beta>0$. There are $0,1,2$ equilibria in $D_{0}, D_{1}, D_{2}$, respectively.

We have

$$
\begin{aligned}
& c \leqslant c^{*} \Longleftrightarrow A_{1}(c) \geqslant A_{2}(c), \\
& c>c^{*} \Longleftrightarrow A_{1}(c)<A_{2}(c) .
\end{aligned}
$$

Therefore, when $c \leqslant c^{*}$, system (6) does not have any endemic equilibrium in this case. If $c>c^{*}$, then system (6) has two equilibria when $A_{1}(c)<A<A_{2}(c)$ and $\Delta>0$; system (6) has a unique endemic equilibrium when $A=A_{2}(c)$; system (6) has a unique endemic equilibrium of multiplicity 2 when $A_{1}(c)<A<A_{2}(c)$ and $\Delta=0$; and system (6) has no equilibrium when $\Delta<0$.

Solving the equation $\Delta=0$ for $A$, one gets two curves

$$
\Gamma_{1}: A=A_{3}(c), \quad \Gamma_{2}: A=A_{4}(c)
$$

in the $(c, A)$ plane, where

$$
\begin{aligned}
& A_{3}(c)=\frac{(d-b \beta)(d+v)+c \beta+2 \sqrt{c \beta(d-b \beta)(d+v)}}{\beta}, \\
& A_{4}(c)=\frac{(d-b \beta)(d+v)+c \beta-2 \sqrt{c \beta(d-b \beta)(d+v)}}{\beta} .
\end{aligned}
$$

For any point of $(c, A) \in \Gamma_{2}$, we have $A<A_{1}(c)$ by simple calculation. The two lines $A=A_{1}(c), A=A_{2}(c)$ joint the curve $\Gamma_{1}: A=A_{3}(c)$ at a point $P\left(A_{1}\left(c^{*}\right), c^{*}\right)$ when $c=c^{*}$. For any $c>c^{*}$, the curve $\Gamma_{1}$ lies above $A=A_{1}(c)$ and under $A=A_{2}(c)$ as shown in Figure 2. In fact, we have $\Delta<0$ for the point $(c, A)$ on $A=A_{1}(c)$ and $\Delta>0$ for the point $(c, A)$ on $A=A_{2}(c)$, and hence the curve $\Gamma_{1}$ lies between the two lines $A=A_{1}(c)$ and $A=A_{2}(c)$ when $c>c^{*}$. As is shown in Figure 2, in the case when $d-b \beta>0$, the curve $A=A_{2}(c)$ and the curve segment $\Gamma_{1}$ subdivide the positive 
cone of the parameters plane $(c, A)$ into three regions $D_{0}, D_{1}$ and $D_{2}$, where

$$
\begin{array}{r}
D_{0}=\left\{(c, A) \mid 0<c \leqslant c^{*}, 0<A \leqslant A_{2}(c)\right\} \\
\cup\left\{(c, A) \mid c>c^{*}, 0<A<A_{3}(c)\right\}, \\
D_{1}=\left\{(c, A) \mid 0<c \leqslant c^{*}, A>A_{2}(c)\right\} \\
\cup\left\{(c, A) \mid c>c^{*}, A \geqslant A_{2}(c)\right\}, \\
D_{2}=\left\{(c, A) \mid c>c^{*}, A_{3}(c)<A<A_{2}(c)\right\} .
\end{array}
$$

For parameter $(c, A)$ in each region, the system has exactly 0,1 , and 2 positive equilibria, respectively. In summary, we have the following theorem regarding the number of endemic equilibria.

Theorem 3. For the model (6), with $c^{*}, A_{1}(c), A_{2}(c)$, and $\mathrm{A}_{3}(c)$ defined as previously mentioned,

(1) if $d-b \beta>0$, as shown in Figure 2, one has the following.

(a) For (c, A) $\in D_{0}$, the model (6) has no equilibrium.

(b) For $(c, A) \in D_{1}$, the model (6) has a unique equilibrium $E^{*}$.

(c) For $(c, A) \in D_{2}$, the model (6) has two equilibria $E_{*}$ and $E^{*}$.

(d) For $(c, A) \in \Gamma_{1}$, when $c>c^{*}, E_{*}$ and $E^{*}$ coalesce at a unique endemic equilibrium of multiplicity 2.

(e) At the point $P\left(A_{1}\left(c^{*}\right), c^{*}\right)$, the model (6) has no endemic equilibrium; the disease-free equilibrium is of multiplicity 2.

(2) On the other hand, if $d-b \beta \leqslant 0$, then

(a) if $A>A_{2}(c)$, there is a unique endemic equilibrium $E^{*}$;

(b) if $A \leqslant A_{2}(c)$, there is no endemic equilibrium.

When exist, $E^{*}\left(h\left(I^{*}\right), I^{*}\right)$ and $E_{*}\left(h\left(I_{*}\right), I_{*}\right)$ are the corresponding equilibria, and

$$
\begin{aligned}
& I^{*}=\frac{1}{2}\left[-a_{1}+\sqrt{\Delta(c, A)}\right], \\
& I_{*}=\frac{1}{2}\left[-a_{1}-\sqrt{\Delta(c, A)}\right] .
\end{aligned}
$$

Now we can prove the global stability of the disease-free equilibrium $E_{0}$ when $(c, A) \in D_{0}$ according to Theorem 3 .

Corollary 4. If $(c, A) \in D_{0}$, then the disease-free equilibrium $E_{0}$ of (6) is globally asymptotically stable.

Proof. Let $\Omega=\{(S, I) \mid S, I \geq 0, S+I \leq A / d\}$. We will prove that $\Omega$ is a positively invariant set of (6). By (6), we have $\left.(d S / d t)\right|_{S=0}=A>0$ and $\left.(d I / d t)\right|_{I=0}=0$. Denote $N(t)=S(t)+I(t)$; then

$$
\left.\frac{d N}{d t}\right|_{N=A / d} \leq A-\left.d N\right|_{N=A / d}=0 .
$$

Hence $\Omega$ is a positively invariant set of (6), and it attracts all the positive orbits of (6) state in $R_{2}^{+}$. We have shown that (6) does not have any positive equilibria when $(c, A) \in$ $D_{0}$. It follows from the Poincaré-Bendixson theorem that no periodic orbits (limit cycle) exist in $\Omega$. Since $\Omega$ is a bounded positively invariant region for the model and $E_{0}$ is the unique equilibrium in $\Omega$, the local stability of $E_{0}$ implies that the $\omega$-limit set of every solution with an initial point in $R_{2}^{+}$is $E_{0}$. Hence, the disease-free equilibrium $E_{0}$ is globally asymptotically stable.

\section{Stability and Bifurcations of the Endemic Equilibria}

In this section, we study the stability and bifurcation of the endemic equilibrium. Evaluating the Jacobian of the model (6) at $E^{*}\left(S^{*}, I^{*}\right)$ gives

$$
J=\left(\begin{array}{cc}
-d-\beta I^{*} & -\beta S^{*} \\
\beta I^{*} & \frac{c I^{*}}{\left(b+I^{*}\right)^{2}}
\end{array}\right) .
$$

Then the characteristic equation about $E^{*}$ is given by

$$
\lambda^{2}+H\left(I^{*}, c\right) \lambda+I^{*} G\left(I^{*}, c\right)=0,
$$

where

$$
\begin{aligned}
& H\left(I^{*}, c\right)=d+\beta I^{*}-\frac{c I^{*}}{\left(b+I^{*}\right)^{2}}, \\
& G(I, c)=\frac{A \beta^{2}}{d+\beta I^{*}}-\frac{c\left(d+\beta I^{*}\right)}{\left(b+I^{*}\right)^{2}} .
\end{aligned}
$$

4.1. Case 1: $\mathbb{R}_{0}>1$. When $\mathbb{R}_{0}>1$, the model (6) has a unique endemic equilibrium $E^{*}$. From (24) to (26), we have the following.

Theorem 5. Suppose $\mathbb{R}_{0}>1$. Then the disease endemic equilibrium $E^{*}$ of $(6)$ is a stable node or focus when $H\left(I^{*}, c\right)>$ $0 ; E^{*}$ is an unstable node or focus when $H\left(I^{*}, c\right)<0$, and $(6)$ has at least one closed orbit in $\Omega ; E^{*}$ is a center of the linear system when $H\left(I^{*}, c\right)=0$.

Proof. Rewrite $G(I, c)$ as

$$
\begin{aligned}
G(I, c) & =\beta(d+\beta I)\left[-\frac{c}{\beta(b+I)^{2}}+\frac{\beta A}{(d+\beta I)^{2}}\right] \\
& =\beta(d+\beta I)\left[\frac{d g(I)}{d I}-\frac{d h(I)}{d I}\right] .
\end{aligned}
$$

Note that $d g\left(I^{*}\right) / d I-d h\left(I^{*}\right) / d I>0$; hence $G\left(I^{*}, c\right)>$ 0 . The unique endemic equilibrium $E^{*}$ is a stable node or focus when $H\left(I^{*}, c\right)>0, E^{*}$ is an unstable node or focus when $H\left(I^{*}, c\right)<0 ; E^{*}$ is a center of the linear system when $H\left(I^{*}, c\right)=0$. If $H\left(I^{*}, c\right)<0$, then $E$ is an unstable node or focus. System (6) has at least one closed orbit in $\Omega$ from the Poincaré-Bendixson Theorem. 
As an example, we fix $A=15000, \beta=0.0005, d=$ $0.02, b=1, v=34$. When $c=30$, then $\mathbb{R}_{0}=5.86$; the model (6) has unique endemic equilibrium $E\left(S^{*}, I^{*}\right)=$ $(68189.645,399.949)$ and $H\left(I^{*}, c\right)=0.14534>0$. From Figure 3(a), we can see that the endemic equilibrium $E^{*}$ is stable. When $c=126.99$, then $\mathbb{R}_{0}=2.33$; the model also has a unique endemic equilibrium $E\left(S^{*}, I^{*}\right)=$ $(68678.432,396.818)$ but $H\left(I^{*}, c\right)=-0.01<0$. From Figure 3(b), we can see that the endemic equilibrium $E^{*}$ is unstable and there must be a stable limit cycle around $E\left(S^{*}, I^{*}\right)$.

From Theorem 5, we know that the positive equilibrium $E^{*}$ of system (6) is a center-type nonhyperbolic equilibrium when $H\left(I^{*}, c\right)=0$. Hence, system (6) may undergo Hopf bifurcation in this case. To determine the stability of the endemic equilibrium and direction of Hopf bifurcation in this case, we must compute the Lyapunov coefficients of the equilibrium. We first translate the endemic equilibrium $E^{*}$ of system (6) to the origin with $x=S-S^{*}, y=I-I^{*}$. Then system (6) in a neighborhood $U$ of the origin can be written as

$$
\begin{gathered}
\frac{d x}{d t}=a_{10} x+a_{01} y+a_{11} x y \\
\frac{d y}{d t}=b_{10} x+b_{01} y+b_{11} x y+b_{02} y^{2}+b_{03} y^{3}+O\left(y^{4}\right),
\end{gathered}
$$

where $a_{10}=-d-\beta I^{*}, a_{01}=-\beta S^{*}, a_{11}=-\beta, b_{10}=$ $\beta I^{*}, b_{01}=c I^{*} /\left(b+I^{*}\right)^{2}, b_{11}=\beta, b_{02}=b c /\left(b+I^{*}\right)^{3}, b_{03}=$ $-b c /\left(b+I^{*}\right)^{4}$ and $O\left(y^{4}\right)$ is the same order infinity. Hence, using the formula of the Lyapunov number $\sigma$ for the focus at the origin of (28) in [13], we have

$$
\begin{gathered}
\sigma=\frac{-3 \pi}{2 a_{01} \Delta^{3 / 2}} \\
\times\left[a_{10} b_{10}\left(a_{11}^{2}+a_{11} b_{02}\right)+a_{10} a_{01}\left(b_{11}^{2}+a_{11} b_{02}\right)\right. \\
\quad-2 a_{10} b_{10} b_{02}^{2}+\left(a_{01} b_{10}-2 a_{10}^{2}\right) b_{11} b_{02} \\
\left.\quad-3 b_{10} b_{03}\left(a_{10}^{2}+a_{01} b_{10}\right)\right],
\end{gathered}
$$

where $\Delta=a_{10} b_{01}-a_{01} b_{10}=I^{*} G\left(I^{*}, c\right)>0$.

By numerical simulation, if we fix $d=0.02, v=$ 26, we know there exist parameter values $(A, b, c, \beta)=$ $(1050,500,128228,0.5)$, which satisfies $\mathbb{R}_{0}=92.93>1$ and $H\left(I^{*}, c\right)=0$ such that $\sigma=-97.09<0$. On the other hand, there exist values $(A, b, c, \beta)=(1050,0.1,357.3097,0.5)$, which satisfy $\mathbb{R}_{0}=7.29>1$ and $H\left(I^{*}, c\right)=0$ too, but $\sigma=0.0015>0$. Therefore, there exists an open set $V_{1}$ in the parameter space $(A, b, c, \beta)$, such that $H\left(I^{*}, c\right)=0, \mathbb{R}_{0}>1$ and $\sigma<0$; that is,

$$
\begin{gathered}
V_{1}=\left\{(A, b, c, \beta): A>0, b>0, c>0, \beta>0, \mathbb{R}_{0}>1,\right. \\
\left.H\left(I^{*}, c\right)=0, \sigma<0\right\} .
\end{gathered}
$$

And there exists another open set $V_{2}$ in the parameter space $(A, b, c, \beta)$, such that $H\left(I^{*}, c\right)=0, \mathbb{R}_{0}>1$ and $\sigma>0$; that is,

$$
\begin{gathered}
V_{2}=\left\{(A, b, c, \beta): A>0, b>0, c>0, \beta>0, \mathbb{R}_{0}>1,\right. \\
\left.H\left(I^{*}, c\right)=0, \sigma>0\right\} .
\end{gathered}
$$

By [13], we have the following theorem.

Theorem 6. Suppose $\mathbb{R}_{0}>1$ and there exists $c_{k}>0$ such that $H\left(I^{*}, c_{k}\right)=0$ and $\left.\left(\partial H\left(I^{*}, c\right) / \partial c\right)\right|_{c=c_{k}} \neq 0$ for the endemic equilibrium $E^{*}$ of the model. If $\sigma \neq 0$, then a curve of periodic solutions bifurcates from $E^{*}$ such that the following happens. (1) Suppose $\left(A, b, c_{k}, \beta\right) \in V_{1}$; the model undergoes a supercritical Hopf bifurcation if $\left.\left(\partial H\left(I^{*}, c\right) / \partial c\right)\right|_{c=c_{k}}<0$ and backward supercritical Hopf bifurcation if $\left.\left(\partial H\left(I^{*}, c\right) / \partial c\right)\right|_{c=c_{k}}>0$. (2) Suppose $\left(A, b, c_{k}, \beta\right) \in V_{2}$; the model undergoes a subcritical Hopf bifurcation if $\left.\left(\partial H\left(I^{*}, c\right) / \partial c\right)\right|_{c=c_{k}}<0$ and backward subcritical Hopf bifurcation if $\left.\left(\partial H\left(I^{*}, c\right) / \partial c\right)\right|_{c=c_{k}}>0$.

4.2. Case 2: $\widehat{\mathbb{R}}_{0}<\mathbb{R}_{0}<1$. When $\widehat{\mathbb{R}}_{0}<\mathbb{R}_{0}<1$, model (6) has two endemic equilibria $E_{*}\left(S_{*}, I_{*}\right)$ and $E^{*}\left(S^{*}, I^{*}\right)$ when $a_{1}<0\left(A>A_{1}(c)\right)$. Their coordinates satisfy (7) and $I_{*}<I^{*}$. In this case, we will discuss the stability of endemic equilibrium, Hopf bifurcation, similarly as we did in the previous subsection. In addition, backward bifurcation will be discussed too.

It is easy to see that the Jacobian matrix (6) at $E_{*}$ and $E^{*}$ are the same as (24) if one replaces $I_{*}$ with $I^{*}$. We have the following result.

Theorem 7. Suppose $\widehat{\mathbb{R}}_{0}<\mathbb{R}_{0}<1$ and $a_{1}<0$. Then the endemic equilibrium $E_{*}$ of (6) is a saddle; $E^{*}$ is a stable node or focus when $H\left(I^{*}, c\right)>0 ; E^{*}$ is an unstable node or focus when $H\left(I^{*}, c\right)<0 ; E^{*}$ is a center of the linear system when $H\left(I^{*}, c\right)=0$.

Proof. Because $d g\left(I_{*}\right) / d I-d h\left(I_{*}\right) / d I<0, d g\left(I^{*}\right) / d I-$ $d h\left(I^{*}\right) / d I>0$, we have $G\left(I_{*}, c\right)<0, G\left(I^{*}, c\right)>0$ from (27). Then $E_{*}$ is a saddle, $E^{*}$ is a stable node or focus when $H\left(I^{*}, c\right)>0, E^{*}$ is an unstable node or focus when $H\left(I^{*}, c\right)<$ 0 , and $E^{*}$ is a center of the linear system when $H\left(I^{*}, c\right)=$ 0 .

Here we give some numerical simulations to show that $H\left(I^{*}, c\right)$ may be positive or negative for different parameters. For example, we fix $A=15000, \beta=0.0005, d=$ $0.02, b=0.01, v=34$. When $c=7.16$, we have $\mathbb{R}_{0}=$ 0.50 . System (6) has two endemic equilibria, $E_{*}\left(S_{*}, I_{*}\right)=$ $(749793.72,0.011)$ and $E^{*}\left(S^{*}, I^{*}\right)=(68075.74,400.69)$. We get $H\left(I^{*}, c\right)=0.20247$. From Figure $3(c), E^{*}$ is locally stable. When $c=201$, we have $\mathbb{R}_{0}=0.019$. System (6) has two endemic equilibria too, $E_{*}\left(S_{*}, I_{*}\right)=$ $(739115.82,0.589)$, and $E^{*}\left(S^{*}, I^{*}\right)=(69059.22,394.41)$. In this case, $H\left(I^{*}, c\right)=-0.29239$. From Figure $3(\mathrm{~d})$, we can see that $E^{*}$ is unstable.

From Theorem 7, we know that the endemic equilibrium $E^{*}$ of system (6) is a center-type nonhyperbolic equilibrium when $H\left(I^{*}, c\right)=0$. Hence, system (6) may undergo Hopf 


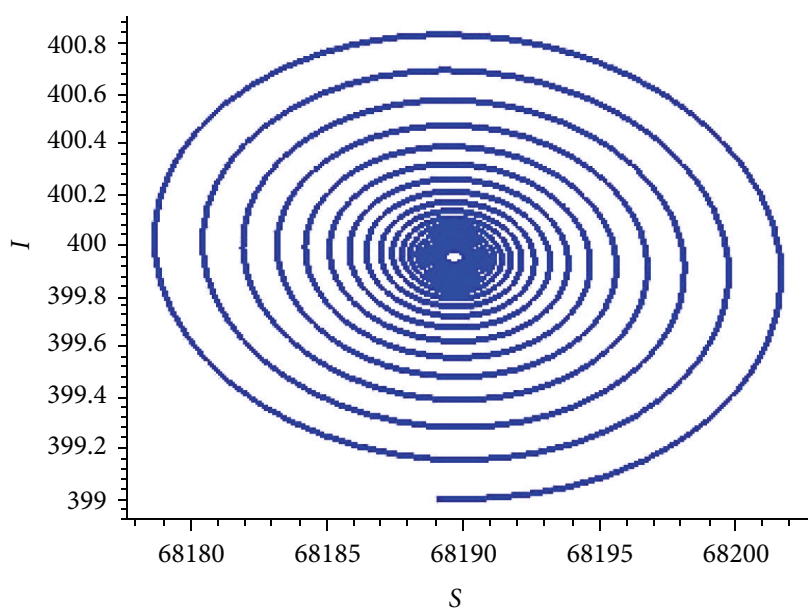

(a) $H\left(I^{*}, c\right)>0$ and $\mathbb{R}_{0}>1$

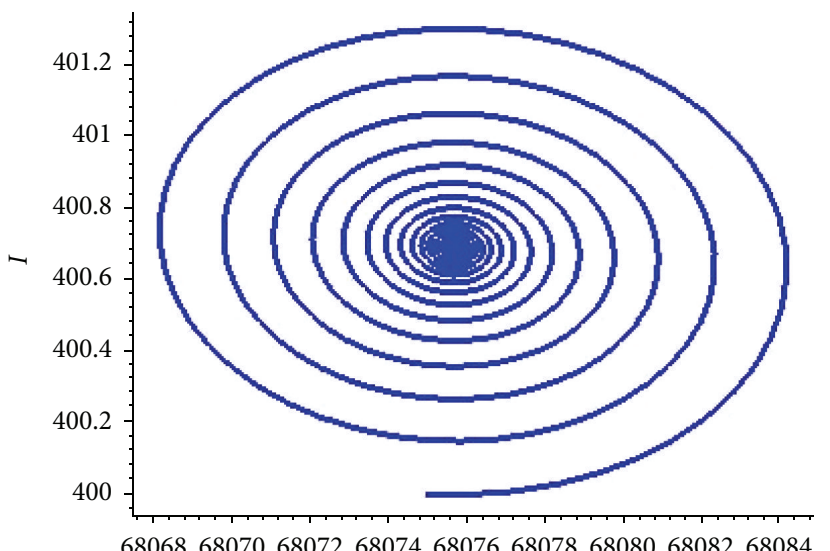

$S$

(c) $H\left(I^{*}, c\right)>0$ and $\mathbb{R}_{0}<1$

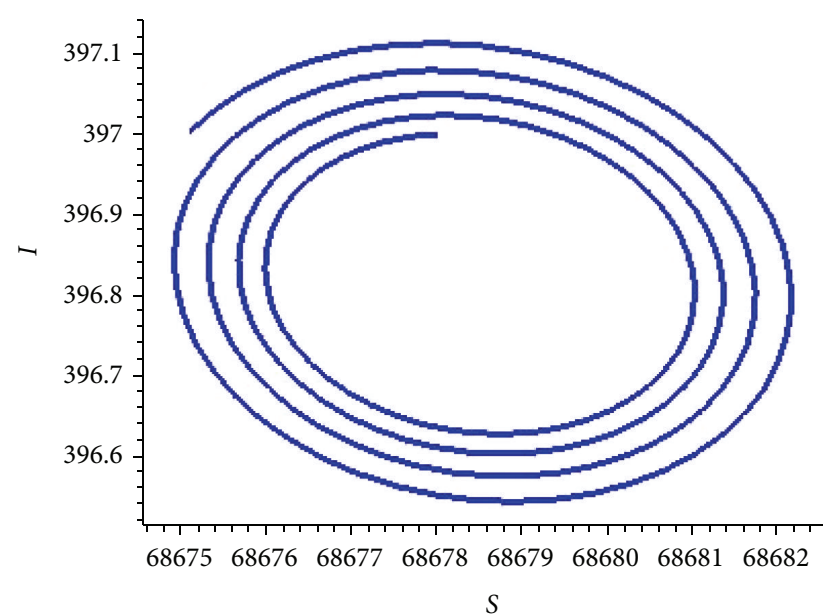

(b) $H\left(I^{*}, c\right)<0$ and $\mathbb{R}_{0}>1$

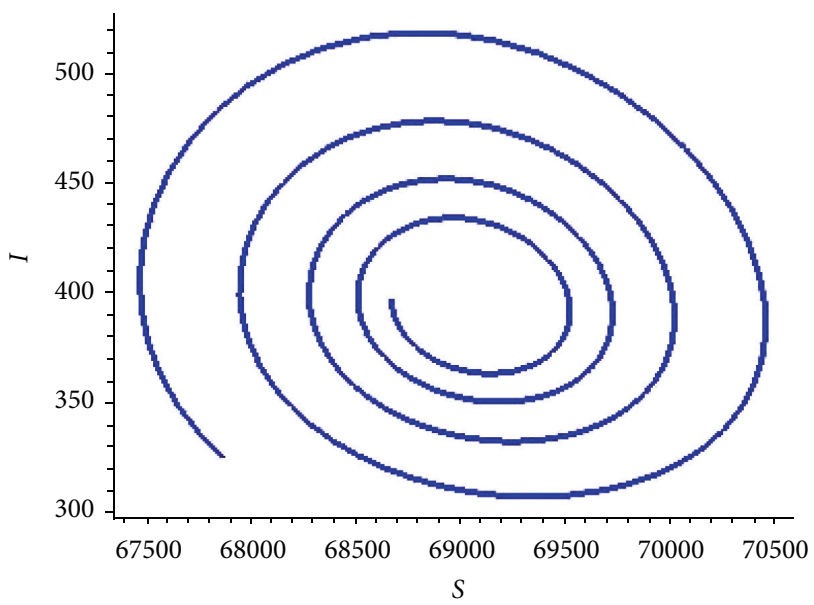

(d) $H\left(I^{*}, c\right)<0$ and $\mathbb{R}_{0}<1$

FIGURE 3: The phase portraits of the model where the directions of trajectories are counterclockwise.

bifurcation in this case. To determine the stability of the endemic equilibrium and direction of Hopf bifurcation in this case, we must compute the Lyapunov coefficients $\sigma$ of the equilibrium $E^{*}$ as we did in Case 1.

Denote nonempty set $V_{3}$ and $V_{4}$ as

$$
\begin{array}{r}
V_{3}=\{(A, b, c, \beta): A>0, b>0, c>0, \beta>0, \\
\left.\widehat{\mathbb{R}}_{0}(c)<\mathbb{R}_{0}<1, H\left(I^{*}, c\right)=0, \sigma<0\right\}, \\
V_{4}=\{(A, b, c, \beta): A>0, b>0, c>0, \beta>0, \\
\left.\widehat{\mathbb{R}}_{0}(c)<\mathbb{R}_{0}<1, H\left(I^{*}, c\right)=0, \sigma>0\right\} .
\end{array}
$$

Theorem 8. Suppose $\widehat{\mathbb{R}}_{0}<\mathbb{R}_{0}<1, a_{1}<0(A>$ $\left.A_{1}(c)\right)$ and there exists $c_{k}>0$ such that $H\left(I^{*}, c_{k}\right)=0$ and $\left.\left(\partial H\left(I^{*}, c\right) / \partial c\right)\right|_{c=c_{k}} \neq 0$ for the endemic equilibrium $E^{*}$ of model (6). If $\sigma \neq 0$, then a curve of periodic solutions bifurcates from $E_{2}$ such that the following happens. (1) Suppose $\left(A, b, c_{k}, \beta\right) \in V_{3}$; then the model undergoes a supercritical Hopf bifurcation if $\left.\left(\partial H\left(I^{*}, c\right) / \partial c\right)\right|_{c=c_{k}}<0$ and backward supercritical Hopf bifurcation if $\left.\left(\partial H\left(I^{*}, c\right) / \partial c\right)\right|_{c=c_{k}}>0$.
(2) Suppose $\left(A, b, c_{k}, \beta\right) \in V_{4}$; then the model undergoes a subcritical Hopf bifurcation if $\left.\left(\partial H\left(I^{*}, c\right) / \partial c\right)\right|_{c=c_{k}}<0$ and backward subcritical Hopf bifurcation if $\left.\left(\partial H\left(I^{*}, c\right) / \partial c\right)\right|_{c=c_{k}}>$ 0 .

According to the aforementioned results, we know that when $d-b \beta>0$ and $c>c^{*}$, besides the basic reproduction number $\mathbb{R}_{0}$, there exists a subthreshold condition for model (3). If $\mathbb{R}_{0} \geq 1$, model (3) has a unique endemic equilibrium which is a node or focus. If $\widehat{\mathbb{R}}_{0}<\mathbb{R}_{0}<1$, model (3) has two endemic equilibria; one is a node or focus and the other is a saddle point. If $\mathbb{R}_{0}=\widehat{\mathbb{R}}_{0}$, model (3) has a unique endemic equilibria of multiplicity two. Otherwise, there is no endemic equilibrium. This situation corresponds to a backward bifurcation (Figure 4). We translate the results into the following theorem.

Theorem 9. If $d-b \beta>0$ and $c>c^{*}$, model (3) undergoes $a$ backward bifurcation at $\mathbb{R}_{0}=1$.

Remark 10. Since the existence of two equilibria and limit cycle under some conditions, model (3) may exhibit two 


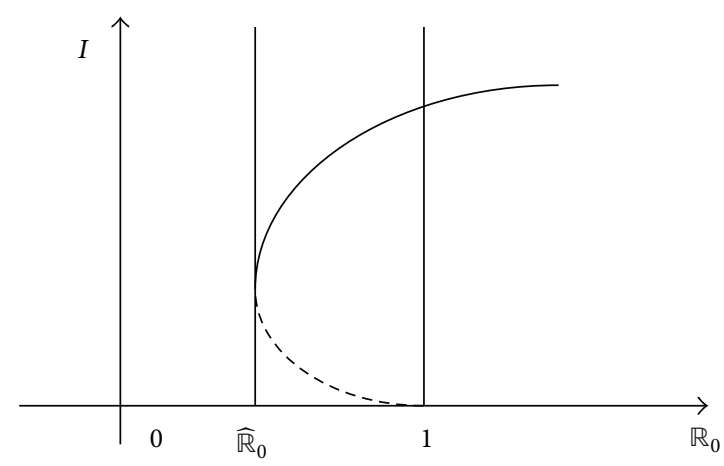

FIgURE 4: A backward bifurcation at $\mathbb{R}_{0}=1$ with a dashed curve for the location of the saddle point and solid curve for the location of the other endemic equilibria.

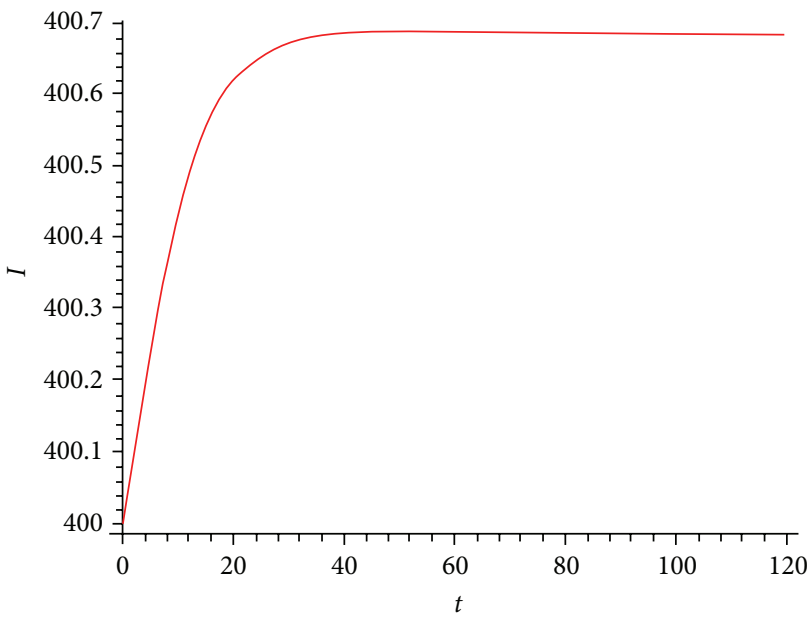

(a) The time course of $I(t)$ when $S(0)=68075, I(0)=400$

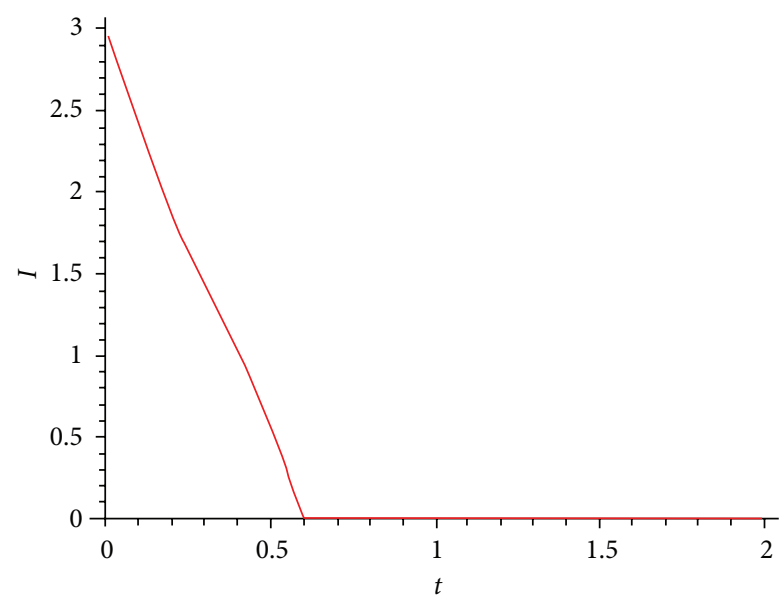

(b) The time course of $I(t)$ when $S(0)=68075, I(0)=3$

FIGURE 5: Different initial states bring different results. The parameters are $A=15000, \beta=0.0005, d=0.02, b=0.01, v=34, c=7.16$, and $\mathbb{R}_{0}=0.50$. The unit of time $t$ is day.

important mathematical phenomena, bistability and periodicity, which can be observed in data for some infectious diseases in history.

\section{Discussion}

In this paper, we proposed a SIR epidemic model with saturation recovery to understand the impact of limited medical resource on infectious disease transmission. Existence of equilibria was obtained under different conditions and their stabilities were analyzed. Bifurcations, including backward bifurcation and Hopf bifurcation, were analyzed too.

The basic reproduction number $\mathbb{R}_{0}$, which gives the expected number of new infections from one infectious individual over the duration of the infectious period, given that all other members of the population are susceptible, in terms of the model parameters, was identified. Our results suggested that for $\mathbb{R}_{0}>1$, the disease-free equilibrium point was unstable while the endemic equilibrium emerged as a unique equilibrium point which implies that disease never dies out epidemiologically. Therefore, bringing the basic reproduction number below 1 is essential. Nevertheless, if $\mathbb{R}_{0}<1$, from
Theorems 2, 3, and 9, the disease-free equilibrium point was not always globally stable and the model may undergo a backward bifurcation, bistability, and periodicity, which implies that reducing the basic reproduction number below 1 is not enough to control the disease in some cases. In order to eradicate the disease, we have a subthreshold number $\widehat{\mathbb{R}}_{0}$ and it is now necessary to reduce $\mathbb{R}_{0}$ to a value less than $\widehat{\mathbb{R}}_{0}$.

$c$, the maximum treatment per unit of time, is related to the maximum treatment capacity in a city or region. Note that $\mathbb{R}_{0}$ is a monotone decreasing function of $c$. Therefore, under the condition of $d-b \beta \leq 0$, increasing $c$ to a value such that $\mathbb{R}_{0}<1$ is sufficient to eliminate the disease. While under the condition of $d-b \beta>0$, increasing $c$ to a value such that $\mathbb{R}_{0}<\widehat{\mathbb{R}}_{0}$ is necessary. Decreasing $c$ to a value at which $\widehat{\mathbb{R}}_{0}<\mathbb{R}_{0}<1$, then whether the disease will break out may depend on the initial conditions. There is a region such that the disease dies out if the initial position lies in this region; otherwise, it tends to an endemic equilibrium $E^{*}$ or a periodic orbit around $E^{*}$. Since the eventual behavior is related to the initial position, this model may be realistic and useful and we should pay more attention to the initial states of the disease (see Figure 5). If $c$ is small enough such that $\mathbb{R}_{0}>1$, 
the disease must break out. Therefore, a sufficient number of sickbeds and other medical resources are very important for the disease control and eradication.

The mathematical analysis of the SIR model has highlighted two important mathematical phenomena observed in data for some infectious diseases: bistability and periodicity. In the bistability phenomenon, the model exhibits multiple endemic equilibria even when $\mathbb{R}_{0}<1$. A clinical study of measles during an endemic in Poland shows that despite high vaccination coverage needed for eradiation since the 1980s, an epidemic of measles with 2255 reported cases occurred between November 1997 and 1998 ([1, 14]). The results of this study confirm that reducing $\mathbb{R}_{0}$ to values less than unity may fail to control the disease. Secondly, the model undergoes oscillatory behavior under certain conditions. The existence of limit cycles confirms such behavior which has been reported in many studies on the dynamics of some infectious diseases such as measles, rubella, and so forth. $([1,10,15])$.

There are many interesting problems related to the models with situation recovery. Some of them for the model will be studied in future. Important dynamical systems features of our models, for example, saddle-node bifurcation, and degenerate Hopf bifurcations, should be discussed.

\section{Acknowledgments}

The authors are grateful to Professor Huaiping Zhu for his valuable comments and suggestions. Hui Wan was supported by NSFC (no. 11201236 and no. 11271196) and the NSF of the Jiangsu Higher Education Committee of China (no. 11KJA110001 and no. 12KJB110012). Jing-an Cui was supported by NSFC (no. 11071011) and Funding Project for Academic Human Resources Development in Institutions of Higher Learning Under the Jurisdiction of Beijing Municipality (no. PHR201107123).

\section{References}

[1] M. E. Alexander and S. M. Moghadas, "Periodicity in an epidemic model with a generalized non-linear incidence," Mathematical Biosciences, vol. 189, no. 1, pp. 75-96, 2004.

[2] X. Shi, J. Cui, and X. Zhou, "Stability and Hopf bifurcation analysis of an eco-epidemic model with a stage structure," Nonlinear Analysis: Theory, Methods \& Applications, vol. 74, no. 4, pp. 1088-1106, 2011.

[3] H. W. Hethcote, "The mathematics of infectious diseases," SIAM Review, vol. 42, no. 4, pp. 599-653, 2000.

[4] Z. Hu, Z. Teng, and H. Jiang, "Stability analysis in a class of discrete SIRS epidemic models," Nonlinear Analysis: Real World Applications, vol. 13, no. 5, pp. 2017-2033, 2012.

[5] J. Pang, J. Cui, and J. Hui, "Rich dynamics of epidemic model with sub-optimal immunity and nonlinear recovery rate," Mathematical and Computer Modelling, vol. 54, no. 1-2, pp. 440-448, 2011.

[6] H. Wan and H. Zhu, "The backward bifurcation in compartmental models for West Nile virus," Mathematical Biosciences, vol. 227, no. 1, pp. 20-28, 2010.
[7] W. Wang, "Backward bifurcation of an epidemic model with treatment," Mathematical Biosciences, vol. 201, no. 1-2, pp. 5871, 2006.

[8] X. Zhang and X. Liu, "Backward bifurcation of an epidemic model with saturated treatment function," Journal of Mathematical Analysis and Applications, vol. 348, no. 1, pp. 433-443, 2008.

[9] http://www.stats.gov.cn/tjsj/qtsj/.

[10] W. Wang and S. Ruan, "Bifurcation in an epidemic model with constant removal rate of the infectives," Journal of Mathematical Analysis and Applications, vol. 291, no. 2, pp. 775-793, 2004.

[11] J. D. Murray, Mathematical Biology, Springer, New York, NY, USA, 1998

[12] P. van den Driessche and J. Watmough, "Reproduction numbers and sub-threshold endemic equilibria for compartmental models of disease transmission," Mathematical Biosciences, vol. 180, pp. 29-48, 2002.

[13] L. Perko, Differential Equations and Dynamical Systems, Springer, New York, NY, USA, 1996.

[14] W. M. Liu, H. W. Hethcote, and S. A. Levin, "Dynamical behavior of epidemiological models with nonlinear incidence rates," Journal of Mathematical Biology, vol. 25, no. 4, pp. 359380, 1987.

[15] J. Lin, V. Andreasen, and S. A. Levin, "Dynamics of influenza A drift: the linear three-strain model," Mathematical Biosciences, vol. 162, no. 1-2, pp. 33-51, 1999. 


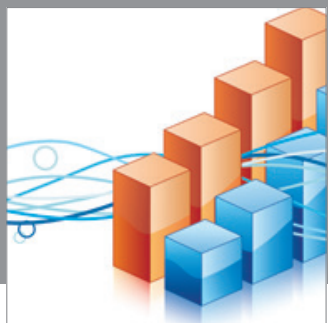

Advances in

Operations Research

mansans

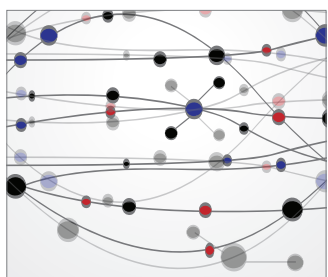

The Scientific World Journal
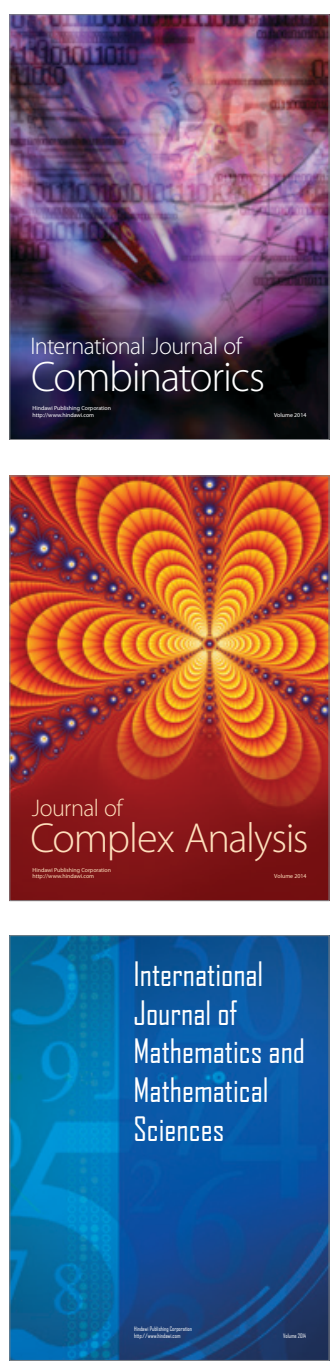
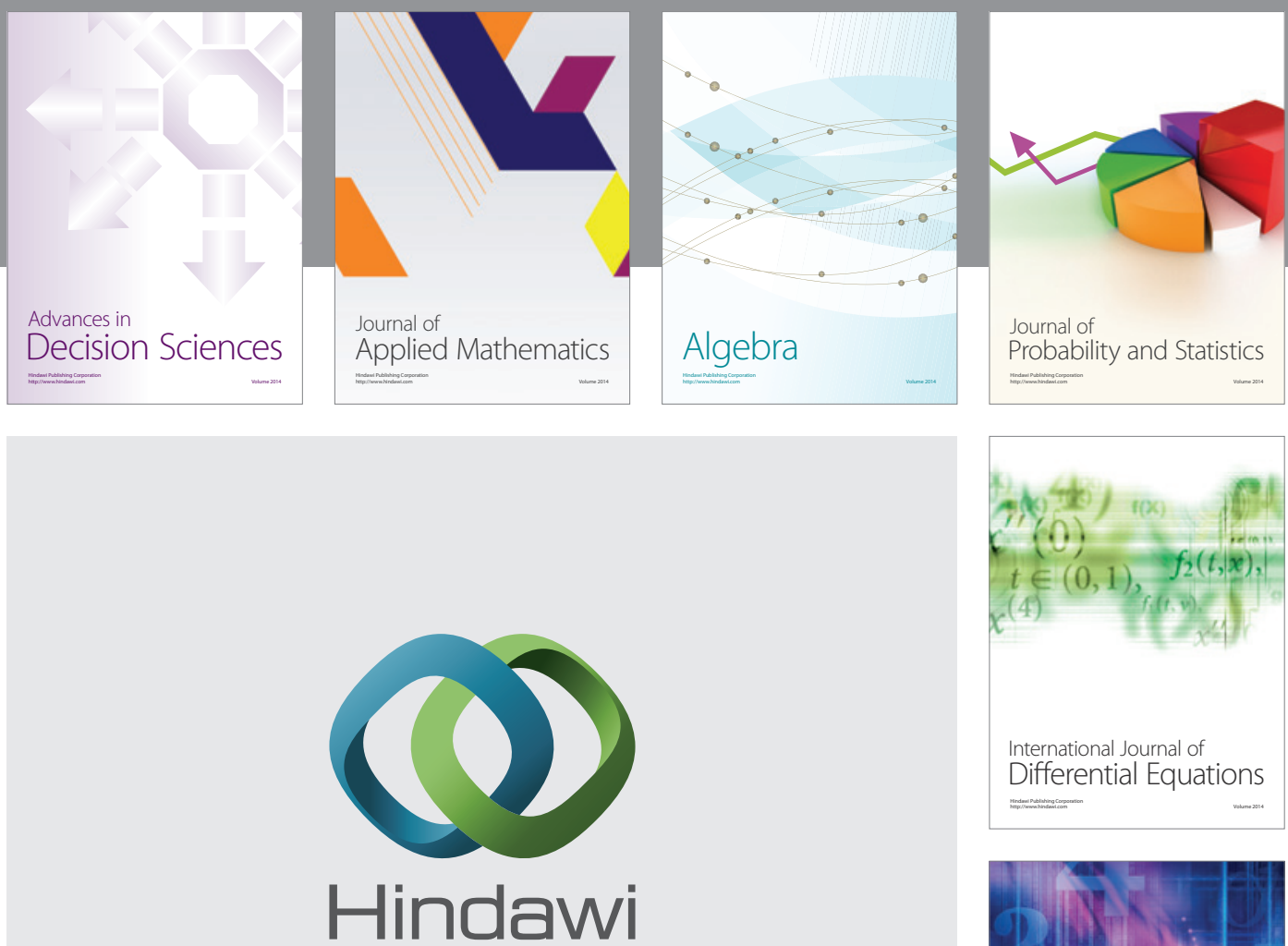

Submit your manuscripts at http://www.hindawi.com
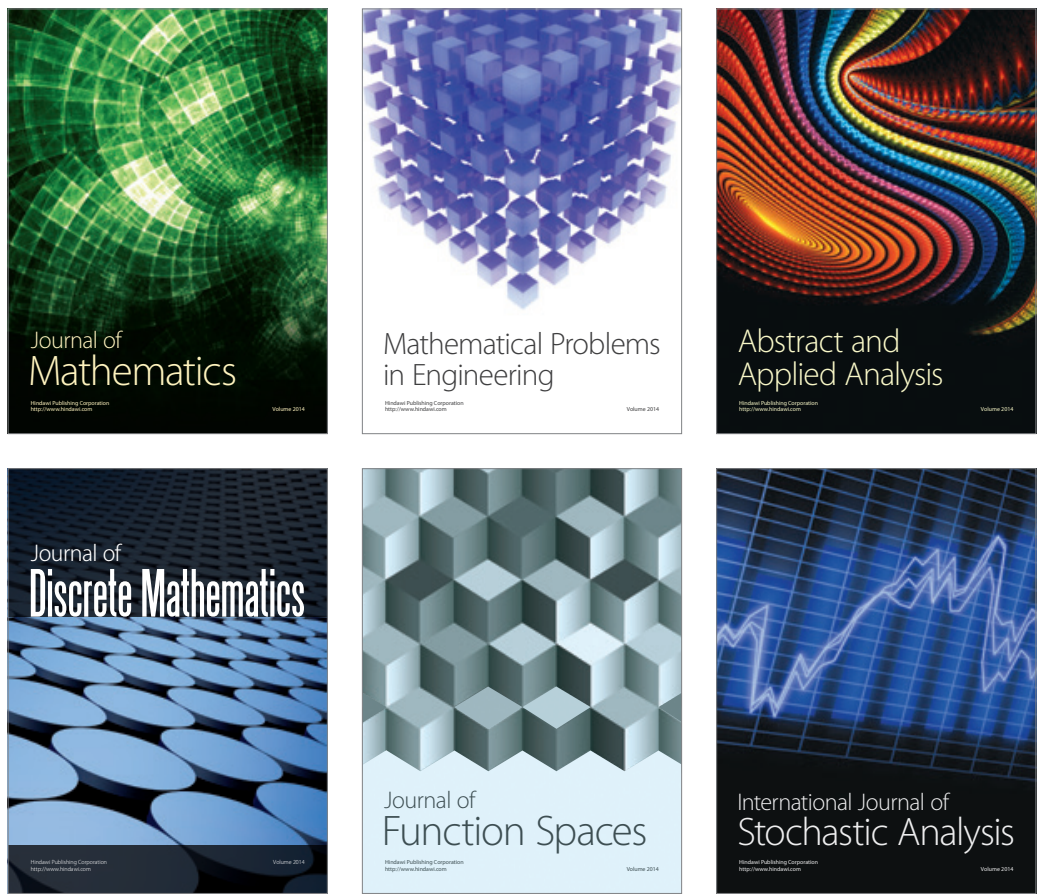

Journal of

Function Spaces

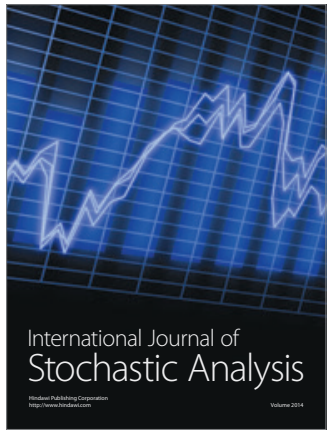

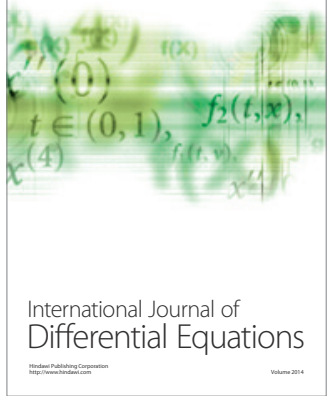
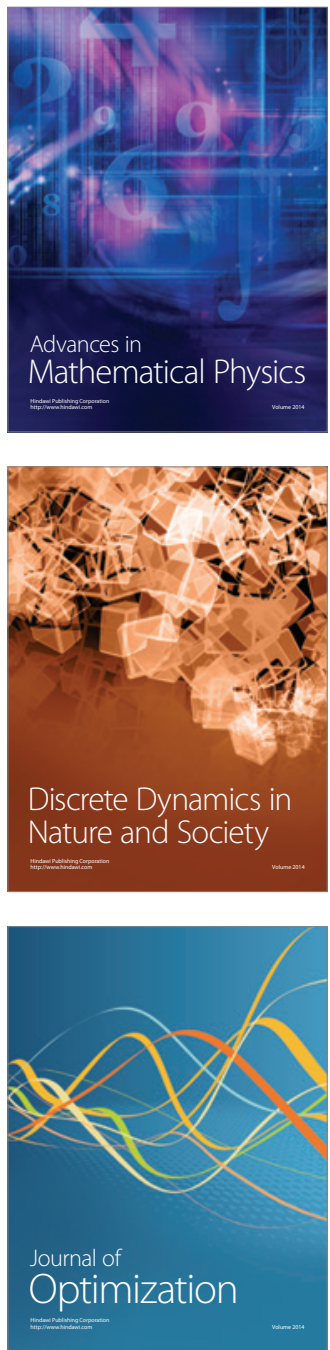